\title{
A CHARACTERIZATION OF LONG EXACT SEQUENCES COMING FROM THE SNAKE LEMMA
}

\author{
JAN ŠŤOVÍČEK
}

AbStract. Given an abelian category, we characterize the long exact sequences of length six which can be obtained from the snake lemma. Equivalently, these are the long exact sequences which arise as the homology of a triangle in the corresponding derived bounded category.

\section{Introduction and preliminaries}

Let $\mathcal{A}$ be an abelian category. We aim to answer the following question (see also the question asked by Deligne at the end of the first part of [6]):

Question. Given a long exact sequence

$$
0 \rightarrow A \stackrel{a}{\longrightarrow} B \stackrel{b}{\longrightarrow} C \stackrel{c}{\longrightarrow} D \stackrel{d}{\longrightarrow} E \stackrel{e}{\longrightarrow} F \rightarrow 0
$$

in $\mathcal{A}$, which conditions must it satisfy so that we can obtain it from the snake lemma as

$$
0 \rightarrow \operatorname{Ker} f_{1} \rightarrow \operatorname{Ker} f_{2} \rightarrow \operatorname{Ker} f_{3} \rightarrow \text { Coker } f_{1} \rightarrow \text { Coker } f_{2} \rightarrow \text { Coker } f_{3} \rightarrow 0,
$$

where $f=\left(f_{1}, f_{2}, f_{3}\right)$ is a suitable homomorphism between short exact sequences in $\mathcal{A}$ ?

This question is an instance of a more general problem. Let $\mathbf{D}^{b}(\mathcal{A})$ be the derived bounded category of $\mathcal{A}$. We ignore the possible set-theoretic difficulties mentioned in $[5, \S 2.2]$, because in what we intend to do with $\mathbf{D}^{b}(\mathcal{A})$ here they do not play any role. Now, one can ask when a long exact sequence

$$
\epsilon: \quad 0 \rightarrow A_{1} \longrightarrow A_{2} \longrightarrow \ldots \longrightarrow A_{n} \rightarrow 0
$$

in $\mathcal{A}$ arises as the homology sequence of a triangle $X \rightarrow Y \rightarrow Z \rightarrow X[1]$ in $\mathbf{D}^{b}(\mathcal{A})$.

For $n=5$ this has been completely resolved by Neeman [6]: the long exact sequence $\epsilon$ is the homology of a triangle if and only if the corresponding class in $\operatorname{Ext}_{\mathcal{A}}^{3}\left(A_{5}, A_{1}\right)$ vanishes. It is worth to mention that Neeman proved more. Namely, if $\epsilon$ comes from a triangle for $n>5$, then the corresponding class in $\mathrm{Ext}_{\mathcal{A}}^{3}$ vanishes for every bit of the form

$$
0 \rightarrow K \longrightarrow A_{i-1} \longrightarrow A_{i} \longrightarrow A_{i+1} \longrightarrow C \rightarrow 0
$$

Received by the editors June 6, 2009.

2000 Mathematics Subject Classification. 18E30 (primary), 18G10 (secondary).

Key words and phrases. long exact sequences, homologies, snake lemma.

The author was supported by the Research Council of Norway through the Storforsk-project "Homological and geometric methods in algebra", and also by the research project MSM 0021620839. 
One may wonder what exactly happens for $n=6$. We have a necessary condition for two classes in $\operatorname{Ext}_{\mathcal{A}}^{3}$, but this is not sufficient as we illustrate in an example at the end of this note. It turns out that we must impose one more condition on a so called Toda bracket. This way, we answer Deligne's question and give a complete solution for $n=6$. Looking back at the very first question about the snake lemma, it is straightforward to see that a long exact sequence of length six comes from the snake lemma if and only if it arises as the homology of a triangle.

In fact, we will tackle a yet more general problem-here we follow the formalism from [6] again. Namely, it is assumed that $\mathcal{A}$ arises as the heart of a t-structure $\left(\mathcal{D}^{\leq 0}, \mathcal{D}^{\geq 0}\right)$ in a triangulated category $\mathcal{T}$. In such a case we have a homological functor $H: \mathcal{T} \rightarrow \mathcal{A}$ sending $X \in \mathcal{T}$ to $\left(X^{\leq 0}\right)^{\geq 0}$, and we let $H^{n}(X)=H(X[n])$. We refer to $[1, \S 1.3]$ for details.

It often happens that there is an exact functor $G: \mathbf{D}^{b}(\mathcal{A}) \rightarrow \mathcal{T}$ extending the identity on $\mathcal{A}$. Then necessarily

$$
\operatorname{Hom}_{\mathbf{D}^{b}(\mathcal{A})}(X, Y[i]) \rightarrow \operatorname{Hom}_{\mathcal{T}}(G X, G Y[i])
$$

is an isomorphism for $X, Y \in \mathcal{A}$ and $i=0,1$. For $i \geq 2$, however, the canonical morphism between the homomorphism groups is far from being an isomorphism in general.

If there is such a functor $G$, we study the problem when a long exact sequence in $\mathcal{A}$ is realized as the homology of a triangle $X \rightarrow Y \rightarrow Z \rightarrow X[1]$ in $\mathcal{T}$ via the homological functor $H: \mathcal{T} \rightarrow \mathcal{A}$. The original problem can be reconstructed by taking $\mathcal{T}=\mathbf{D}^{b}(\mathcal{A})$ together with the canonical t-structure $\left(\mathcal{D}^{\leq 0}, \mathcal{D}^{\geq 0}\right)$, and putting $G=\mathbf{1}_{\mathbf{D}^{b}(\mathcal{A})}$.

For formulating the result, we will need the concept of a Toda bracket. It was first used in [8] and the definition was extended in [3], in both cases in a topological context. Here, we give a purely algebraic definition:

Definition 1. Let $\mathcal{T}$ be a triangulated category and

$$
X \stackrel{x}{\longrightarrow} Y \stackrel{y}{\longrightarrow} Z \stackrel{z}{\longrightarrow} W
$$

be three composable morphisms in $\mathcal{T}$. Consider the following triangle in $\mathcal{T}$ :

$$
Z \longrightarrow V \longrightarrow Y[1] \stackrel{y[1]}{\longrightarrow} Z[1]
$$

Then the Toda bracket $\langle z, y, x\rangle$ is defined as the set of all morphisms $X[1] \rightarrow W$ which can be expressed as the composition of some $g: X[1] \rightarrow V$ and $f: V \rightarrow W$ making the following diagram commutative in $\mathcal{T}$ :

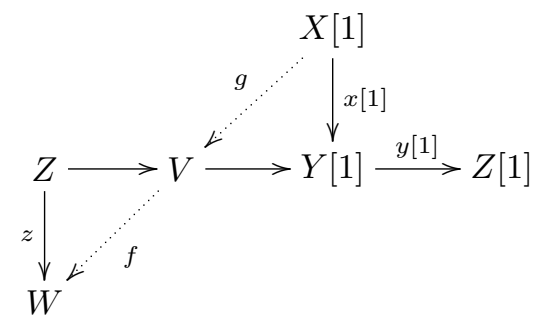


It not difficult to see from the definition that $\langle z, y, x\rangle$ is either empty or a coset of the subgroup $z \operatorname{Hom}(X[1], Z)+\operatorname{Hom}(Y[1], W) x[1]$ of $\operatorname{Hom}(X[1], W)$. Moreover, $\langle z, y, x\rangle \neq \emptyset$ if and only if

$$
X \stackrel{x}{\longrightarrow} Y \stackrel{y}{\longrightarrow} Z \stackrel{z}{\longrightarrow} W
$$

is a chain complex, that is $z y=0=y x$.

We will mostly be interested in whether $0 \in\langle z, y, x\rangle$. This is by far not automatically satisfied even if $\langle z, y, x\rangle$ is non-empty. A simple way of generating examples of this phenomenon is described in [2, §3, pg. 219]; we will use this idea in the example at the end of this note.

\section{The main result}

Our goal here is to prove the following statement:

Theorem 2. Let $\mathcal{A}$ be the heart of a t-structure in a triangulated category $\mathcal{T}$ and assume that there is an exact functor $G: \mathbf{D}^{b}(\mathcal{A}) \rightarrow \mathcal{T}$ extending the identity on $\mathcal{A}$. Let further

$$
0 \rightarrow A \stackrel{a}{\longrightarrow} B \stackrel{b}{\longrightarrow} C \stackrel{c}{\longrightarrow} D \stackrel{d}{\longrightarrow} E \stackrel{e}{\longrightarrow} F \rightarrow 0
$$

be an exact sequence in $\mathcal{A}$. Denote by $K, L, M$ be the images of $B \rightarrow C, C \rightarrow D$ and $D \rightarrow E$, respectively, and denote as follows the morphisms in $\mathcal{T}$ coming from the short exact subsequences of $(*)$ :

$$
F \stackrel{\alpha}{\longrightarrow} M[1] \stackrel{\beta}{\longrightarrow} L[2] \stackrel{\gamma}{\longrightarrow} K[3] \stackrel{\delta}{\longrightarrow} A[4] .
$$

Then the following are equivalent:

(1) There exist a triangle $X \rightarrow Y \rightarrow Z \rightarrow X[1]$ in $\mathcal{T}$ whose homology is isomorphic to $(*)$.

(2) $0 \in\langle\delta, \gamma \beta, \alpha\rangle$.

Remark 3. Note that $0 \in\langle\delta, \gamma \beta, \alpha\rangle$ automatically implies that $\delta \gamma \beta=0=\gamma \beta \alpha$. That is, the image under $G: \mathbf{D}^{b}(\mathcal{A}) \rightarrow \mathcal{T}$ of the corresponding classes in $\operatorname{Ext}_{\mathcal{A}}^{3}(M, A)$ and $\operatorname{Ext}^{3}(F, K)$ coming from $(*)$ vanishes.

If we already know that $\delta \gamma \beta=0=\gamma \beta \alpha$, then the condition $0 \in\langle\delta, \gamma \beta, \alpha\rangle$ can be restated as follows. Let $W$ fit into the triangle $D[-1] \rightarrow W \rightarrow C \stackrel{c}{\rightarrow} D$. Then there is a natural triangle

$$
K[2] \longrightarrow W[2] \longrightarrow M[1] \stackrel{\gamma \beta}{\longrightarrow} K[3]
$$

coming from the exact sequence $0 \rightarrow K \longrightarrow C \stackrel{c}{\longrightarrow} D \longrightarrow M \rightarrow 0$. Moreover, we know that the morphisms $F \rightarrow M[1]$ and $K[2] \rightarrow A[3]$ coming from $(*)$ factorize (non-uniquely) through $W[2] \rightarrow M[1]$ and $K[2] \rightarrow W[2]$, respectively:

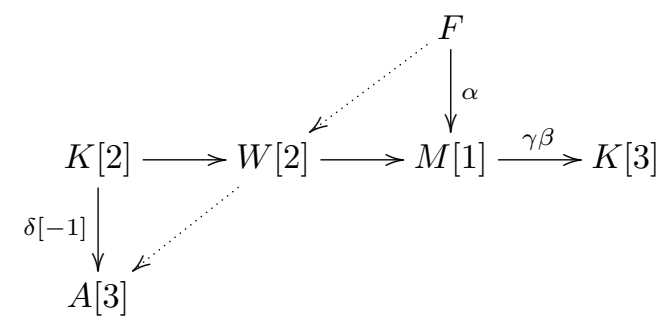


The assertion in Theorem 2(2) is then equivalent to being able to choose the factorization so that the composition $F \rightarrow W[2] \rightarrow A[3]$ vanishes.

Proof. (1) $\Longrightarrow(2)$. Assume there is a triangle

$$
X \stackrel{x}{\longrightarrow} Y \stackrel{y}{\longrightarrow} Z \stackrel{z}{\longrightarrow} X[1]
$$

in $\mathcal{T}$ whose homology is $(*)$; say $H^{0}(X)=A$. Then the main result of [6] says that $\delta \gamma \beta=0=\gamma \beta \alpha$ and hence $\langle z, y, x\rangle \neq \emptyset$, but this will also follow from our argument directly.

We need to prove more, namely that $0 \in\langle z, y, x\rangle$. To do this, note first that we can without loss of generality assume that $X, Y, Z \in \mathcal{D}^{\geq-1} \cap \mathcal{D}^{\leq 1}$. If not we can replace the original triangle $X \rightarrow Y \rightarrow Z \rightarrow X[1]$ by:

$$
\left(X^{\leq 1}\right) \geq 0 \stackrel{\left(x^{\leq 1}\right)^{\geq 0}}{\longrightarrow}\left(Y^{\leq 1}\right) \geq 0 \stackrel{y^{\prime}}{\longrightarrow} Z^{\prime} \stackrel{z^{\prime}}{\longrightarrow}\left(X^{\leq 1}\right) \geq 0[1] .
$$

The long exact sequence of homologies remains unchanged by [1, Proposition 1.3.5], and we have $\left(X^{\leq 1}\right)^{\geq 0},\left(Y^{\leq 1}\right)^{\geq 0}, Z^{\prime} \in \mathcal{D}^{\geq-1} \cap \mathcal{D}^{\leq 1}$.

Let us next consider the truncations of $X$ with respect to $\left(\mathcal{D}^{\leq 0}, \mathcal{D}^{\geq 0}\right)$ :

$$
X^{\leq 0} \longrightarrow X \longrightarrow X^{\geq 1} \longrightarrow\left(X^{\leq 0}\right)[1]
$$

An easy computation of homologies shows that $X^{\leq 0} \cong A$ and $X^{\geq 1} \cong D[-1]$. Note that here we need the fact that $X \in \mathcal{D}^{\geq-1} \cap \mathcal{D}^{\leq 1}$, since in general it is perfectly possible that there are non-zero objects in $\mathcal{T}$ whose homologies with respect to $\left(\mathcal{D}^{\leq 0}, \mathcal{D}^{\geq 0}\right)$ all vanish. By forming a homotopy push-out of

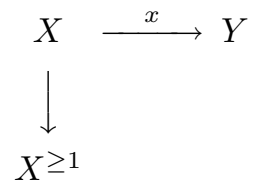

we subsequently obtain the following diagram with triangles in rows and columns:

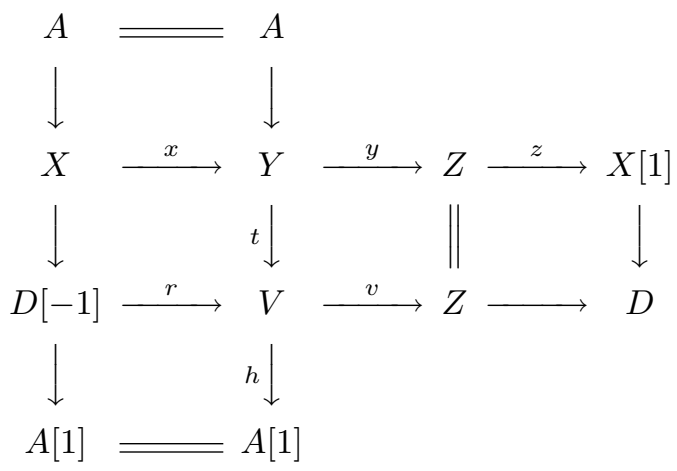

Similarly, one easily computes that $Z^{\leq 0} \cong C$ and $Z^{\geq 1} \cong F[-1]$. The truncation with respect to the t-structure gives a triangle

$$
F[-2] \longrightarrow C \longrightarrow Z[-1]
$$


and by constructing a homotopy pull-back of

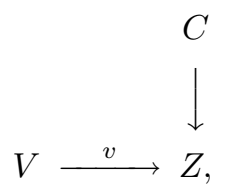

one obtains a diagram:

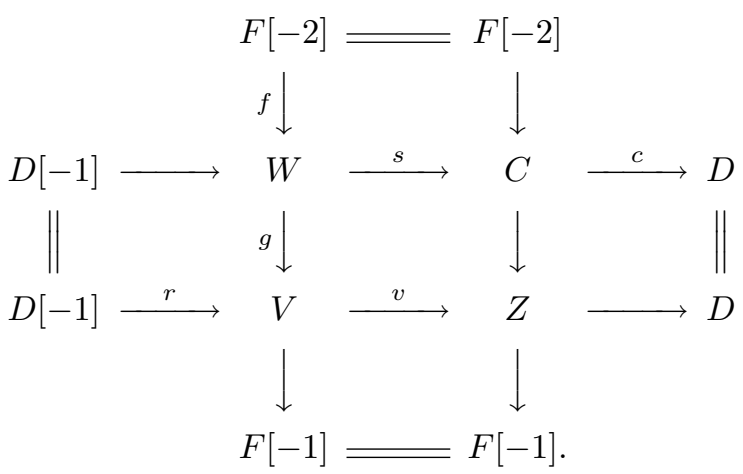

Clearly, the composition

$$
F \stackrel{f[2]}{\longrightarrow} W[2] \stackrel{(h g)[2]}{\longrightarrow} A[3],
$$

is zero, where the morphisms $f, g$ and $h$ come from diagrams $(\dagger)$ and $(\ddagger)$.

All we have to show now is that the morphisms $f[2]: F \rightarrow W[2]$ and $(h g)[2]$ : $W[2] \rightarrow A[3]$ fit up to isomorphism the definition of the Toda bracket. More precisely, consider the triangle

$$
K[2] \stackrel{\epsilon}{\longrightarrow} W[2] \stackrel{\zeta}{\longrightarrow} M[1] \stackrel{\gamma \beta}{\longrightarrow} K[3] .
$$

We will prove that there are automorphisms $\phi$ and $\psi$ of $F$ and $A[3]$, respectively, such that the following equalities hold:

$$
\begin{aligned}
\alpha & =\zeta \circ f[2] \circ \phi, \\
\delta[-1] & =\psi \circ(h g)[2] \circ \epsilon .
\end{aligned}
$$

For the first equality, note that $W \leq 0 \cong K \cong V \leq 0$ and $g \leq 0: W \leq 0 \rightarrow V \leq 0$ is an isomorphism. Taking into account that $W^{\geq 1} \cong M[-1]$ and $V^{\geq 1} \cong E[-1]$, we get the following commutative diagram with triangles in rows:

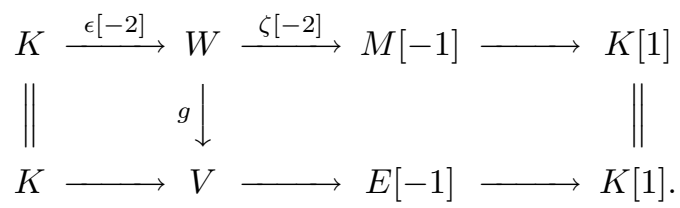

We can choose the morphisms in the upper row as stated since the triangle there is a truncation triangle of $W$ with respect to the t-structure and as such it is unique up to a unique isomorphism. By [5, Lemma 1.4.3], we can complete this diagram with a morphism $M[-1] \rightarrow E[-1]$ such that the resulting morphism of triangles can be completed to an octahedron. Note that there is in fact only one morphism $M[-1] \rightarrow$ $E[-1]$ which completes the diagram, namely $g^{\geq 1}$, and it is up to isomorphism just 
the shift of the monomorphism $M \rightarrow E$ in $\mathcal{A}$ coming from (*) in the statement of Theorem 2. We can summarize our findings in the following diagram with triangles in rows and columns:

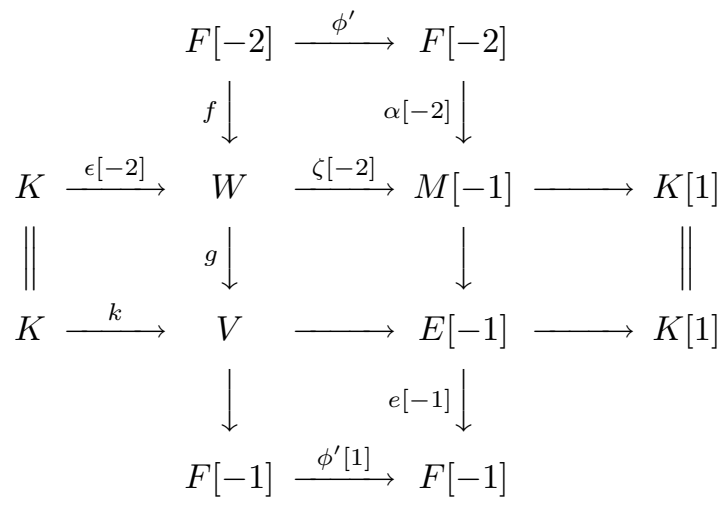

We do not know whether $\phi^{\prime}$ is the identity in general, but it certainly is an isomorphism since $(\Delta)$ is an instance of the octahedral axiom. The desired equality appears in the top square in the diagram when taking $\phi=\left(\phi^{\prime}[2]\right)^{-1}$.

Let us finally prove that $\delta[-1]=\psi \circ(h g)[2] \circ \epsilon$ for some automorphism $\psi$. As before, one readily checks that $Y^{\geq 1} \cong E[-1] \cong V^{\geq 1}$ and $t^{\geq 1}: Y^{\geq 1} \rightarrow V^{\geq 1}$ is an isomorphism (see diagram $(\dagger$ ) for the morphism $t: Y \rightarrow V$ ), and that one can obtain a diagram

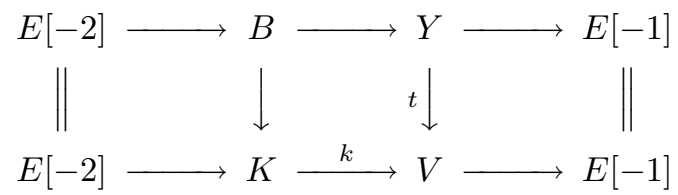

which admits a completion to an octahedron. Again, the morphism $B \rightarrow K$ in the diagram is up to isomorphism nothing else than the epimorphism in $\mathcal{A}$ coming from (*) in the statement of Theorem 2 . Therefore, we obtain the following commutative diagram with triangles in rows and columns:

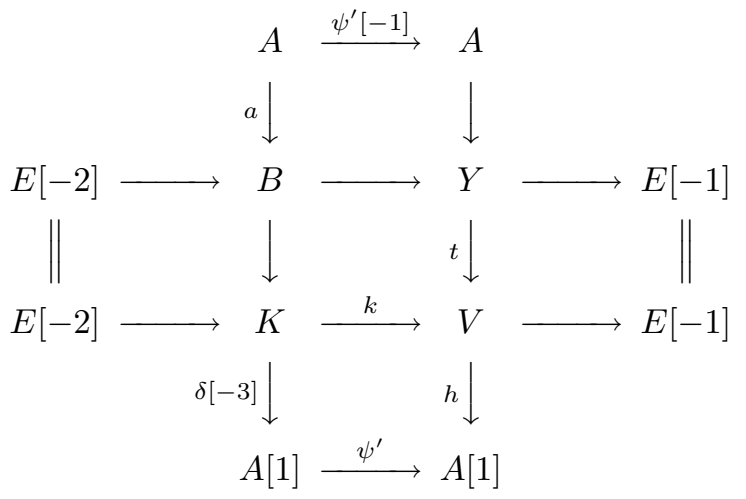

Since $(\Xi)$ is an instance of the octahedral axiom, $\psi^{\prime}$ is an isomorphism. From the bottom square we see that $\delta[-3]=\left(\psi^{\prime}\right)^{-1} h k$, and by combining this with the equality coming from the leftmost square of diagram $(\Delta)$, we get $\delta[-3]=\left(\psi^{\prime}\right)^{-1} h g \circ \epsilon[-2]$. 
The equality we wanted to prove is obtained by shifting this by two and putting $\psi=\left(\psi^{\prime}[2]\right)^{-1}$. This concludes the proof of $(1) \Longrightarrow(2)$.

$(2) \Longrightarrow(1)$. This follows basically by retracing the steps. Assume we have an exact sequence as in $(*)$ satisfying $(2)$. Let us denote the morphisms constructed in Remark 3 by $f^{\prime}: F \rightarrow W[2]$ and $g^{\prime}: W[2] \rightarrow A[3]$. First we put $f=f^{\prime}[-2]$ and form the triangles

$$
D[-1] \longrightarrow W \stackrel{s}{\longrightarrow} C \stackrel{c}{\longrightarrow} D
$$

and

$$
F[-2] \stackrel{f}{\longrightarrow} W \stackrel{g}{\longrightarrow} V \longrightarrow F[-1]
$$

Next we form diagram $(\ddagger)$ as a homotopy pushout of

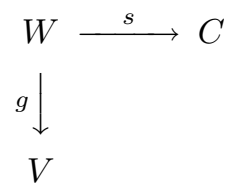

Because $g^{\prime}[-2] \circ f=0$ by assumption, there is a morphism $h: V \rightarrow A[1]$ such that $h g=g^{\prime}[-2]$. Considering the triangle

$$
A \longrightarrow Y \stackrel{t}{\longrightarrow} V \stackrel{h}{\longrightarrow} A[1]
$$

this allows us to form diagram $(\dagger)$ as a homotopy pull-back of

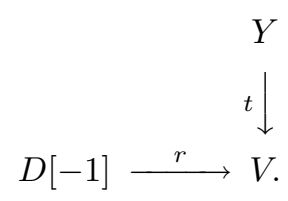

Now we can define the triangle

$$
X \stackrel{x}{\longrightarrow} Y \stackrel{y}{\longrightarrow} Z \stackrel{z}{\longrightarrow} X[1]
$$

for condition (1) as the second row of $(\dagger)$ and we must prove that its homology is indeed isomorphic to $(*)$.

Also here we only reverse the arguments. We know that the homology of triangle $(\sharp)$ is isomorphic to

$$
0 \rightarrow K \longrightarrow C \stackrel{c}{\longrightarrow} D \longrightarrow M \rightarrow 0 .
$$

By examining columns in diagram $(\Delta)$, which in this case can be constructed with $\phi^{\prime}=\mathbf{1}_{F[-2]}$, starting with the commutative square

$$
\begin{array}{cc}
F[-2] & F[-2] \\
f \downarrow & \alpha[-2] \downarrow \\
W & \stackrel{\zeta[-2]}{\longrightarrow} \\
& M[-1],
\end{array}
$$

one immediately sees that

$$
0 \rightarrow H^{1}(W) \stackrel{H^{1}(g)}{\longrightarrow} H^{1}(V) \longrightarrow H^{1}(F[-1]) \rightarrow 0
$$


is a short exact sequence corresponding to $\alpha: F \rightarrow M[1]$. Then it easily follows from diagram ( $\ddagger$ ) that the homology of the triangle $D[-1] \stackrel{r}{\rightarrow} V \stackrel{v}{\rightarrow} Z \rightarrow D$ is precisely

$$
0 \rightarrow K \longrightarrow C \stackrel{c}{\longrightarrow} D \stackrel{d}{\longrightarrow} E \stackrel{e}{\longrightarrow} F \rightarrow 0 .
$$

A similar argument shows that the homology of triangle $(\diamond)$ is the long exact sequence $(*)$ from the statement of the theorem, which concludes the proof.

\section{An example}

It is relatively easy to construct an exact sequence of length six which does not come from the snake lemma. Namely, let $R$ be any ring admitting a module $M$ of projective dimension at least 4 and consider the beginning of the projective resolution

$$
0 \rightarrow \Omega^{4} M \longrightarrow P_{3} \longrightarrow P_{2} \longrightarrow P_{1} \longrightarrow P_{0} \longrightarrow M \rightarrow 0 .
$$

Such a sequence certainly cannot come from the snake lemma because it has a nonzero class in $\operatorname{Ext}_{R}^{4}\left(M, \Omega^{4} M\right)$.

We are going to construct an example with finer properties. Namely, if $k$ is a field and $R=k[x] /\left(x^{3}\right)$, we will construct an exact sequence

$$
0 \rightarrow A \stackrel{a}{\longrightarrow} B \stackrel{b}{\longrightarrow} C \stackrel{c}{\longrightarrow} D \stackrel{d}{\longrightarrow} E \stackrel{e}{\longrightarrow} F \rightarrow 0
$$

such that in the notation of Theorem 2 :

(1) $\delta \gamma \beta=0=\gamma \beta \alpha$,

(2) $0 \notin\langle\delta, \gamma \beta, \alpha\rangle$.

This will also show that the necessary condition given by Neeman $[6]$ is not sufficient.

In order to verify the properties of our forthcoming example, we need a little more theory. If $R$ is a finite dimensional self-injective algebra over a field $k$, we denote by mod- $R$ the category of all finite dimensional modules and by mod- $R$ the corresponding stable category modulo projectives-injectives. It is well known that $\bmod -R$ has a natural triangulated structure with the shift functor being the cosyzygy functor $\Omega^{-}$ (see for example $[4, \S 1])$. Moreover, there is a close link between $\mathbf{D}^{b}(\bmod -R)$ and $\underline{\bmod }-R$ :

Proposition 4. Let $R$ be a finite dimensional self-injective algebra over a field. Then there is an exact functor $Q: \mathbf{D}^{b}(\bmod -R) \rightarrow \underline{\bmod }-R$ such that

(1) The following diagram with the obvious functors is commutative:

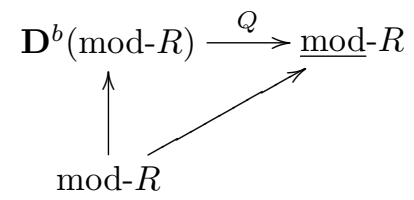

(2) The induced homomorphisms

$$
\operatorname{Hom}_{\mathbf{D}^{b}(\bmod -R)}(X, Y[i]) \longrightarrow \underline{\operatorname{Hom}}\left(Q X, \Omega^{-i} Q Y\right)
$$

are isomorphisms for each $X, Y \in \bmod -R$ and $i \geq 1$. 
Proof. (1). This follows from (the proof of) [7, Theorem 2.1]. Namely, if perf- $R$ stands for the full triangulated subcategory of $\mathbf{D}^{b}(\bmod -R)$ formed by the perfect complexes, then $Q$ is obtained as a composition of the localization functor

$$
Q^{\prime}: \mathbf{D}^{b}(\bmod -R) \longrightarrow \mathbf{D}^{b}(\bmod -R) / \text { perf- } R
$$

with a quasi-inverse to the natural functor (which is a triangle equivalence by [7]):

$$
\underline{\bmod }-R \longrightarrow \mathbf{D}^{b}(\bmod -R) / \text { perf- } R \text {. }
$$

(2). This follows by considering the isomorphisms

$$
\operatorname{Hom}_{\mathbf{D}^{b}(\bmod -R)}(X, Y[i]) \cong \operatorname{Ext}_{R}^{i}(X, Y) \cong \underline{\operatorname{Hom}}\left(X, \Omega^{-i} Y\right)
$$

and taking into account the construction of $Q$.

Now we can construct the example.

Example 5. Let $k$ be a field and $R=k[x] /\left(x^{3}\right)$. Let $Q: \mathbf{D}^{b}(\bmod -R) \rightarrow \underline{\bmod }-R$ be a functor as in Proposition 4. Let us further consider the unique composition series

$$
0 \subseteq S \subseteq N \subseteq R
$$

of $R$. It is well known that $S, N$ and $R$ are up to isomorphism the only indecomposables in mod- $R$. The non-split exact sequence $0 \rightarrow S \longrightarrow N \longrightarrow S \rightarrow 0$ in mod- $R$ yields the following triangle in mod- $R$ :

$$
S \longrightarrow N \longrightarrow S \longrightarrow N\left(=\Omega^{-} S\right) \text {. }
$$

This can be rewritten as:

$$
S \stackrel{\alpha^{\prime}}{\longrightarrow} \Omega^{-} S \stackrel{\eta^{\prime}}{\longrightarrow} \Omega^{-3} N \stackrel{\delta^{\prime}}{\longrightarrow} \Omega^{-4} N
$$

Obviously $\delta^{\prime} \eta^{\prime}=0=\eta^{\prime} \alpha^{\prime}$ and one easily checks that $0 \notin\left\langle\delta^{\prime}, \eta^{\prime}, \alpha^{\prime}\right\rangle$, for example by using the comment on contractible triangles from [2, §3, pg. 219]. By Proposition 4, we can find morphisms

$$
S \stackrel{\alpha}{\longrightarrow} S[1] \stackrel{\eta}{\longrightarrow} N[3] \stackrel{\delta}{\longrightarrow} N[4]
$$

in $\mathbf{D}^{b}(\bmod -R)$ such that the images of $\alpha, \eta, \delta$ under $Q$ are $\alpha^{\prime}, \eta^{\prime}, \delta^{\prime}$, respectively. It follows immediately that $\delta \eta=0=\eta \alpha$ and $0 \notin\langle\delta, \eta, \alpha\rangle$.

One can construct exact sequences in mod- $R$ corresponding to $\alpha, \eta$ and $\delta$ in a standard way by using injective coresolutions and pull-backs:

$$
\begin{array}{ll}
\alpha: & 0 \rightarrow S \longrightarrow N \longrightarrow S \rightarrow 0, \\
\eta: & 0 \rightarrow N \longrightarrow R \longrightarrow N \longrightarrow S \rightarrow 0, \\
\delta: & 0 \rightarrow N \longrightarrow S \oplus R \longrightarrow N \rightarrow 0 .
\end{array}
$$

We leave the details for the reader. If we now splice these three sequences, we get an exact sequence of legth six with the required properties:

$$
0 \rightarrow N \longrightarrow S \oplus R \longrightarrow R \longrightarrow N \longrightarrow N \longrightarrow S \rightarrow 0 .
$$

That is, this sequence satisfies the necessary condition from [6] (the non-empty Toda bracket), but it still cannot be obtained from the snake lemma because the Toda bracket does not contain the zero morphism. 


\section{References}

[1] A. A. Bĕlinson, J. Bernstein, and P. Deligne, Faisceaux pervers, in Analysis and topology on singular spaces, I (Luminy, 1981), Vol. 100 of Astérisque, 5-171, Soc. Math. France, Paris (1982).

[2] M. Bökstedt and A. Neeman, Homotopy limits in triangulated categories, Compositio Math. 86 (1993), no. 2, 209-234.

[3] J. M. Cohen, The decomposition of stable homotopy, Ann. of Math. (2) 87 (1968) 305-320.

[4] D. Happel, Triangulated categories in the representation theory of finite-dimensional algebras, Vol. 119 of London Mathematical Society Lecture Note Series, Cambridge University Press, Cambridge (1988), ISBN 0-521-33922-7.

[5] A. Neeman, Triangulated categories, Vol. 148 of Annals of Mathematics Studies, Princeton University Press, Princeton, NJ (2001), ISBN 0-691-08685-0; 0-691-08686-9.

[6] - Long exact sequences coming from triangles, in Proceedings of the 39th Symposium on Ring Theory and Representation Theory, 23-29, Symp. Ring Theory Represent. Theory Organ. Comm., Yamaguchi (2007).

[7] J. Rickard, Derived categories and stable equivalence, J. Pure Appl. Algebra 61 (1989), no. 3, 303-317.

[8] H. Toda, Composition methods in homotopy groups of spheres, Annals of Mathematics Studies, No. 49, Princeton University Press, Princeton, N.J. (1962).

Institutt for matematiske fag, NTNU, N-7491 Trondheim, Norway

E-mail address: stovicek@math.ntnu.no 